\title{
THE NUMERICAL SOLUTION OF
}

\section{ELLIPTIC AND PARABOLIC PARTIAL}

DIFFERENTIAL EQUATIONS WITH BOUNDARY

SINGULARITIES

by

J.CRANK and R.M. FURZELAND 


\begin{abstract}
A general numerical method is described for the solution of linear elliptic and parabolic partial differential equations in the presence of boundary singularities. The method is suitable for use with either a finite-difference or finite element scheme. Modified approximations for the derivatives are developed using the local analytical form of the singularity. General guidelines are given showing how the local analytical form can be found and how the modified approximations can be developed for many problems of mathematical physics. These guidelines are based on the reduction of the differential equation to the form $\Delta \mathrm{u}=\mathrm{gu}+\mathrm{f}$. The potential problem treated by Motz and Woods is taken as a numerical example. The numerical results compare favourably with those obtained by other techniques.
\end{abstract}





\section{Introduction}

The problem of boundary singularities in the numerical solution of elliptic and parabolic partial differential equations has received a great deal of attention. These singularities arise when sudden changes occur either in the direction of the boundary, as at a re-entrant corner, or they may be associated with mixed boundary conditions. Such singularities are found in a wide variety of physical problems e.g. stress analysis in regions with cracks, discontinuities, point sources etc. [8,14,43], flow around an obstacle [18], seepage through a dam [1], heat flow, diffusion or potential problems in regions with re-entrant corners, electrodes, heat sources or sinks [3-7,11-15,19-25,27,30-39,42].

Analytical solutions based on separable_variable or integral transform techniques are available for infinite or semi-infinite regions with relatively simple governing equations (usually Laplace's) and boundary conditions [25,27,30], However such solutions are, in general, difficult to obtain for finite regions with more complicated equations and boundary conditions and so numerical solutions are considered.

It will be shown later how the field gradients become unbounded at such singularities. This property means that standard numerical approximations using a uniform mesh for the derivatives in the governing differential equation are highly inaccurate at and near the singularity. For example, consider the usual five-point finite-difference approximation $\Delta^{(5)} \mathrm{u}(\mathrm{x}, \mathrm{y})$ for the Laplacian $\Delta \phi$,

$$
\Delta^{(5)} u=\Delta u+\frac{2 h^{2}}{4 !}\left[\frac{\partial^{4} u}{\partial x^{4}}+\frac{\partial^{4} u}{\partial y^{4}}\right]+\ldots,
$$

where $\mathrm{h}$ is the mesh length in both the $\mathrm{x}$ and $\mathrm{y}$ directions. The usual assumption that terms of order $\mathrm{h}^{2}$ are negligible is no longer true because unbounded derivatives exist near the singularity. For similar reasons the methods of deferred correction and deferred approach to 
the limit are difficult to apply near such singularities. Although the errors die away with distance from the singularity (the rate at which they die away depending on the neighbouring boundary conditions), their effects spread throughout the entire region. This is called the 'pollution effect' by Babuska and Aziz [2].

Special numerical schemes have been devised to obtain accurate solutions without a large amount of computation, the two most popular methods being:

I - mesh refinement near the singularity $[7,12,19,32,36,39]$,

II - modified approximations to the governing differential equation and its solution near the singularity, e.g. as in the Motz method $[3-6,8,11,14,15,17,21,35,37,42,43]$.

Either method may be part of, and dovetails readily into, a global finite-difference or finite element scheme. In method II, the standard approximations near the singularity are replaced by modified approximations based on the local analytical form of the singularity. Such forms require some prior analysis but usually are readily available in the form of an asymptotic expansion by separable-variable [4,17,28,43] or complex variable [20,22,40,41] techniques. Although method I is computationally inefficient relative to method II, since the order of the matrix is increased, it is a viable alternative in that no knowledge of the form of the singularity is required and any symmetry or banding present is preserved. Either method can be used if more than one singularity is present as long as the singularities are far enough apart, or the mesh is fine enough, for the effect of one singularity on another to be negligible.

Alternative methods based on conformal transformations [23,24] modified integral equations [31], modified collocation [29], power series [22], dual series [38], and removal of the singularity [34,40-42] have been proposed. The most promising of these is the conformal transformation method which has proved to be highly accurate and efficient for the solution of elliptic problems in simply-connected polygonal regions with general mixed boundary conditions. The method 
is limited to differential equations which remain invariant under conformal transformations, e.g. Laplace's equation in Cartesian co-ordinates.

Application of the above methods has been restricted, for the most part, to equations of the form

$$
\sum_{i=1}^{n} K_{i} \frac{\partial^{2} u}{\partial x_{i}} 2=g u+f
$$

where $\mathrm{K}_{\mathrm{i}}=0,1$ or -1 , and $\mathrm{u}, \mathrm{g}$, f are functions of $\mathrm{x}_{\mathrm{i}}$ only. Courant and Hilbert [10], with later work by Sankar [28], have given a general technique for reducing the general linear differential equation of second order,

$$
\sum_{i, k=1}^{n} A_{i k} \frac{\partial^{2} U}{\partial^{X_{i} X_{k}}}+\sum_{i=1}^{n} B_{i} \frac{\partial U}{\partial X_{i}}+C U+D=0,
$$

$\mathrm{A}_{\mathrm{ik}}, \mathrm{B}_{\mathrm{i}}, \mathrm{C}, \mathrm{D}$ are functions of $\mathrm{X}_{\mathrm{i}}$ alone, to the simple form (1.2). This is valuable in that available techniques for equations of the form (1.2) can now be used. In particular, the general separablevariable technique of Fox and Sankar [17], which gives the asymptotic expansions needed by method II for equations of the form (1.2), can now be used. Also, work is in progress on extending the conformal transformation techniques to equations of the form (1.2), sea [19], and so this method in conjunction with the above reduction, will then be capable of dealing with general equations of the form (1.3). Further, the invariance of the left-hand side of (1.2) under conformal transformations means that method II can be extended to cover curved boundaries by conformal transformations to straight line boundaries.

In the following sections a new way of developing the modified approximations for method II for differential equations of elliptic and parabolic type is given. The method differs from available methods of type II in that modified approximations for the derivatives in the governing equation are developed and take the place of the algebraic 
equations for the solution values as originally used by Motz [21]. General equations of the form (1.3) are first reduced to the form (1.2) in order to take advantage of the points mentioned above and to keep the number of derivative approximations to a minimum. The method is applied to the potential problem of Motz [21] and Woods [42]. First, a discussion of how the separable-variable technique can be used to find the local form of the singularity for a wide range of practical problems of mathematical physics is given.

\section{Local analytical forms for boundary singularities}

In this section local analytical forms for the solution of general linear second order differential equations of elliptic and parabolic type for general boundary conditions are developed. The solutions are obtained in the form of asymptotic expansions using separablevariable techniques rather than complex variable ones since the latter, although useful for analytical properties such as existence $[20,22,40,41]$, are more complicated to apply in practice.

Consider, first, equation (1.2) in the Cartesian co-ordinates (x,y) with a singularity at the point $(a, b)$ on the boundary. In local polar co-ordinates $(\rho, \theta)$ centred on the singularity, $x=a+\rho \cos \theta$ and $\mathrm{y}=\mathrm{b}+\rho \sin \theta$, equation (1.2) with $\mathrm{k}_{1}=\mathrm{k}_{2}=1$ is

$$
\frac{\partial^{2} \mathrm{u}}{\partial \rho^{2}}+\frac{1}{\rho} \frac{\partial \mathrm{u}}{\partial \rho}+\frac{1}{\rho^{2}} \frac{\partial^{2} \mathrm{u}}{\partial \theta^{2}}=\mathrm{gu}+\mathrm{f} .
$$

If a separable-variable solution of the form

$$
u(\rho, \theta)=R(\rho) \Theta(0)
$$

is sought then, for the case $\mathrm{f}=\mathrm{g}=0$, one obtains the following harmonic forms of the singularity corresponding to the point where two straight-line boundary segments $\theta=0$ and $\theta=\omega$ meet with homogeneous Dirichlet, Neumann or mixed boundary conditions: 
(i) for $\mathbf{u}=0$ on $\theta=0$ and $\theta=\omega$,

$$
\mathrm{u}(\rho, \theta)=\sum_{\mathrm{k}=1}^{\infty} \mathrm{c}_{\mathrm{k}} \rho^{\frac{\mathrm{k} \pi}{\omega}} \sin \frac{\mathrm{k} \pi}{\omega} \theta, \mathrm{c}_{\mathrm{k}} \text { are arbitrary constants, }
$$

(ii) for $\frac{\partial u}{\partial \theta}=0$ on $\theta=0$ and $\theta=\omega$,

$$
\mathrm{u}(\rho, \theta)=\sum_{\mathrm{k}=0}^{\infty} \mathrm{c}_{\mathrm{k}} \rho^{\frac{\mathrm{k} \pi}{\omega}} \cos \frac{\mathrm{k} \pi}{\omega} \theta,
$$

(iii) for $\frac{\partial u}{\partial \theta}=0$ on $\theta=0$ and $u=0$ on $\theta=\omega$,

$$
\mathrm{u}(\rho, \theta)=\sum_{\mathrm{k}=0}^{\infty} \mathrm{c}_{\mathrm{k}} \rho^{\left(\mathrm{k}+\frac{1}{2}\right) \frac{\pi}{\omega}} \cos \left(\mathrm{k}+\frac{1}{2}\right) \frac{\pi}{\omega} \theta .
$$

If the conditions in (iii) are interchanged then the cosine function in (2.5) is replaced with the sine function. The above forms are only valid provided that any other singularities are far enough away for their effect to be assumed negligible. For cases (i) and (ii), by considering the $k=1$ terms, it can be seen that $\frac{\partial u}{\partial \rho}$ is infinite at $\rho=0$ for $\omega>\pi$ (re-entrant corner). Similarly for (iii), by considering the $k=0$ term, $\frac{\partial u}{\partial \rho}$ is infinite at $\rho=0$ for $\omega>\pi / 2$, i.e. even for obtuse $\omega$.

Corresponding results for non-zero g in (2.1) of the form

$$
\mathrm{g}(\rho, \theta)=\sum_{\mathrm{n}=0}^{\infty} \mathrm{g}_{\mathrm{n}}(\theta) \rho^{\mathrm{n}}
$$

for the more general boundary conditions

$$
\left.\begin{array}{c}
u=F(\rho) \quad=\sum_{n=0}^{\infty} f_{n} \rho^{n+\beta}, \quad \beta \geq 0 \\
\frac{1}{\rho} \frac{\partial u}{\partial \theta}=H(\rho)=\sum_{n=0}^{\infty} h_{n} \rho^{n+\gamma}, \quad \gamma \geq 0
\end{array}\right\} \text { on either } \theta=0 \text { or } \theta=\omega,
$$


are given in Fox and Sankar [17]. Following Fox [16] these results may be summarised as

$$
u=\phi_{0}(\rho, \theta)+\sum_{i=1}^{\infty} c_{i} \phi_{i}(\rho, \theta)
$$

where the $c_{i}$ are arbitrary constants. Each $\phi(\rho, \theta)$ is a linear combination of terms like

$$
\rho^{\alpha+\mathrm{i}} \mathrm{A}_{\alpha, \mathrm{i}}(0) \text { or } \rho^{\alpha+\mathrm{i}}\left\{(\operatorname{In} \rho) \mathrm{A}_{\alpha, \mathrm{i}}(\theta)+\mathrm{B}_{\alpha, \mathrm{i}}(\theta)\right\} \text {, }
$$

where $\alpha$ is found by fitting (2.7) and depends on $n$; $A$, B are trigonometric terms obtained from a sequence of ordinary differential equations with constant coefficients e.g. for the first of (2.9),

$$
\left.\begin{array}{l}
\mathrm{A}_{\alpha, 0}^{\prime \prime},+\alpha^{2} \mathrm{~A}_{\alpha, 0}=0 \\
\mathrm{~A}_{\alpha, 1}^{\prime \prime}+(\alpha+1)^{2} \mathrm{~A}_{\alpha, 1}=0 \\
\mathrm{~A}_{\alpha, \mathrm{m}+2}^{\prime \prime}+(\alpha+\mathrm{m}+2)^{2} \mathrm{~A}_{\alpha, \mathrm{m}+2}=-\sum_{\mathrm{j}=0}^{\mathrm{m}} \mathrm{g}_{\mathrm{m}-\mathrm{j}} \mathrm{A}_{\alpha, \mathrm{j}}, \mathrm{m}=0,1,2 \ldots
\end{array}\right\}
$$

The presence of a non-zero f in (2.1) merely adds known terms to (2.8).

The corresponding problem

$$
\frac{\partial^{2} u}{\partial x^{2}}+\frac{\partial^{2} u}{\partial y^{2}}-\frac{\partial u}{\partial t}=g u+f
$$

for boundary conditions of the form (2.7) has been treated by Bell C4] by seeking solutions of the form

$$
\mathrm{u}=\mathrm{e}^{-\lambda^{2} \mathrm{t}} \mathrm{R}(\rho) \Theta(\theta)+\mathrm{W}(\rho, \theta)
$$

where $W(\rho, \theta)$ represents the steady state solution. 
For more general elliptic and parabolic equations of the form (1.3) the above techniques could be extended to cover the first or cross derivative terms e.g. see Sankar [28] or Zak [43]. However the presence of such terms, particularly if non-constant coefficients are also present, considerably complicates the corresponding polar co-ordinate equation and the sequence of equations for the functions of $\theta$ in (2.9). A more convenient way of proceeding is to reduce the general equation (1.3) to the simpler form (1.2) by transformations of the co-ordinates $X_{i}$ to $x_{i}$ and of the unknown function $U$ to $u$ and then seek a separable-variable solution. First, (1.3) is reduced to the canonical (normal) form

$$
\sum_{i=1}^{n} K_{i} \frac{\partial^{2} U}{\partial x_{i}^{2}}+\sum_{i=1}^{n} b_{i} \frac{\partial U}{\partial x_{i}}=c U+d,
$$

where $\mathrm{K}_{\mathrm{i}}=0,1$ or -1 , and $\mathrm{b}_{\mathrm{i}}, \mathrm{c}, \mathrm{d}$ are functions of $\mathrm{x}_{\mathrm{i}}$, by the transformation of co-ordinates

$$
x_{i}=x_{i}\left(X_{1}, X_{2}, \ldots, X_{n}\right), \quad i=1,2, \ldots, n
$$

In this form equation (1.3) can readily be classified, see Riz [26]. Equation (1.3) is elliptic if in (2.13) all the $\mathrm{K}_{\mathrm{i}}$ are non-zero and of the same sign (either +1 or -1 ), hyperbolic if all the $K_{i}$ are non-zero and one $K_{i}$ is of opposite sign to the rest, parabolic if one or more of the $\mathrm{K}_{\mathrm{i}}$ are zero.

The condition for this change of co-ordinate system can conveniently be expressed in terms of tensor calculus for the n-dimensional space $\mathrm{V}_{\mathrm{n}}$ corresponding to the co-ordinates. The condition is that the space $V_{n}$ be flat for which the necessary and sufficient condition is that the Riemann-Christoffel (or curvature) tensor be identically zero, see Sankar [28]. In the two dimensional case there is just one condition since the rest of the components of this tensor are zero. Also, in this case, the simpler algebra enables the condition to be derived from first principles e.g. in the elliptic case with $\mathrm{D}=$ $\mathrm{B}_{1}=\mathrm{B}_{2}=\mathrm{C}=0, \mathrm{~A}_{12}=\mathrm{A}_{21}$ in (1.3), it gives rise to the Beltrami 
differential equations for $\mathrm{x}_{1}$ and $\mathrm{x}_{2}$

$$
\left.\begin{array}{l}
\frac{\partial \mathrm{x}_{2}}{\partial \mathrm{x}_{1}}=\frac{1}{\mathrm{~W}}\left[\mathrm{~A}_{12} \frac{\partial \mathrm{x}_{1}}{\partial \mathrm{X}_{1}}+\mathrm{A}_{22} \frac{\partial \mathrm{x}_{1}}{\partial \mathrm{X}_{2}}\right] \\
\frac{\partial \mathrm{x}_{2}}{\partial \mathrm{X}_{2}}=-\frac{1}{\mathrm{~W}}\left[\mathrm{~A}_{11} \frac{\partial \mathrm{x}_{1}}{\partial \mathrm{X}_{1}}+\mathrm{A}_{12} \frac{\partial \mathrm{x}_{1}}{\partial \mathrm{X}_{2}}\right]
\end{array}\right\} \text { where } \mathrm{W}=\sqrt{\mathrm{A}_{11} \mathrm{~A}_{12}-\mathrm{A}_{12}^{2}}
$$

The derivation and solution of (2.15) is given in Courant and Hilbert [10, Ch.III and p. 350].

Equation (2.13) is now reduced to the form (1.2), viz.

$$
\sum_{i=1}^{n} K_{i} \frac{\partial^{2} u}{\partial x^{2}}=g u+f, \quad K_{i}=0,1 \text { or }-1
$$

by using the exponential transformation of Sankar [28]

$$
\mathrm{u}=\mathrm{U} \exp \left\{\frac{1}{2} \sum_{\mathrm{i}=1}^{\mathrm{n}} \int \mathrm{b}_{\mathrm{i}} \mathrm{dx} \mathrm{x}_{\mathrm{i}}\right\},
$$

which removes the first derivative terms in (2.13). A similar transformation was given by Courant and Hilbert [10,p.183] for the case when the $b_{i}$ are constant. In the parabolic case this transformation corresponds to the removal of the exponential time term as in (2.12). The following examples illustrate the use of the transformations (2.14) and (2.17):

(i) Reduction of Tricomi's equation

$$
\frac{\partial^{2} \mathrm{U}}{\partial \mathrm{x}^{2}}+\mathrm{x} \frac{\partial^{2} \mathrm{U}}{\partial \mathrm{y}^{2}}=0
$$

for the elliptic case $x>0$, to the canonical form (2.13) by the transformation of co-ordinates (page 162, Courant and Hilbert)

$$
\mathrm{x}_{1}=-\mathrm{x}^{3 / 2}, \quad \mathrm{x}_{2}=\frac{3}{2} \mathrm{y}
$$


giving

$$
\frac{\partial^{2} \mathrm{U}}{\partial \mathrm{x}_{1}^{2}}+\frac{1}{3 \mathrm{x}_{1}} \frac{\partial \mathrm{U}}{\partial \mathrm{x}_{1}}+\frac{\partial^{2} \mathrm{U}}{\partial \mathrm{x}_{2}^{2}}=0
$$

Tricomi's equation is of special interest in high velocity gas flows [9].

(ii) Reduction of

$$
\frac{\partial^{2} \mathrm{U}}{\partial \mathrm{x}_{1}^{2}}+\frac{\mathrm{k}}{\mathrm{x}_{1}} \frac{\partial \mathrm{U}}{\partial \mathrm{x}_{1}}+\frac{\partial^{2} \mathrm{U}}{\partial \mathrm{x}_{2}^{2}}=0
$$

to the form (2.16) by the use of transformation of unknown function

$$
\mathrm{u}=\mathrm{x}_{1}^{\mathrm{k} / 2} \mathrm{U}
$$

giving

$$
\frac{\partial^{2} \mathrm{u}}{\partial \mathrm{x}_{1}^{2}}+\frac{\partial^{2} \mathrm{u}}{\partial \mathrm{x}_{2}^{2}}=-\frac{\mathrm{k}}{2 \mathrm{x}_{1}^{2}}\left(\frac{1}{2} \mathrm{k}-1\right)
$$

The case $k>0$ represents the family of axially symmetric problems discussed in Crank and Furzeland [11]. The case $\mathrm{k}<0$ represents axially symmetric flow problems where $U$ is the stream function. In the parabolic case where the right-hand side of (2.21) contains a $\frac{\partial U}{\partial t}$ term, the transformation (2.21) can still be used and just gives a $\frac{\partial \mathrm{U}}{\partial \mathrm{t}}$ term in $(2.23)$

(iii) More complicated equations may require both transformation of coordinates and functions to obtain the form (2.16), e.g.

$$
\mathrm{X}_{1}^{2} \frac{\partial^{2} \mathrm{U}}{\partial \mathrm{X}_{1}^{2}}+\mathrm{X}_{1} \frac{\partial \mathrm{U}}{\partial \mathrm{X}_{1}}+\left(1+\mathrm{X}_{1}^{2}\right) \frac{\partial^{2} \mathrm{U}}{\partial \mathrm{X}_{2}^{2}}=0
$$

which arises in the vortex theory of screw propellors needs both transformations, see Fox and Sankar [17]. Tricomi's equation can be reduced to (2.23) if both transformations (2.19) and (2.22) are used. 
10.

Boundary conditions of the third kind,

$$
\frac{\partial \mathrm{U}}{\partial \mathrm{x}_{\mathrm{i}}}+\mathrm{P}_{\mathrm{i}} \mathrm{U}=\mathrm{Q}_{\mathrm{i}}
$$

where $\mathrm{P}_{\mathrm{i}}, \mathrm{Q}_{\mathrm{i}}$ are functions of $\mathrm{x}_{\mathrm{i}}, \mathrm{i}=1,2, \ldots, \mathrm{n}$, are harder to deal with since separable-variable solutions of the form (2.9), and which fit (2.25), are harder to find. In such cases the exponential trans formation

$$
\mathrm{u}=\mathrm{U} \exp \left\{\int P_{i} d x_{i}\right\}
$$

can be used to reduce (2.25) to

$$
\frac{\partial \mathrm{u}}{\partial \mathrm{x}_{\mathrm{i}}}=0
$$

however this transformation adds corresponding first derivative terms to the governing equation. Thus for third boundary value problems the separable-variable solution has to be found from equations of the form (2.13), which contains first derivative terms, rather than from equations of the form (2.16). Conversely, if one is treating problems with derivative boundary conditions, and the differential equation (2.13) contains first derivative terms with respect to the same variable that appears in the derivative boundary condition, then the exponential transformation (2.17) cannot be used since it would transform the boundary condition to the type $(2.25)$.

Non-linear differential equations or non-linear boundary conditions cannot be dealt with using the techniques of this section unless some transformation or approximation to linear form is used. For example the compressible flow equation for high velocity gas flow

$$
\frac{\partial}{\partial X_{1}}\left(\rho \frac{\partial U}{\partial X_{1}}\right)+\frac{\partial}{\partial X_{2}}\left(\rho \frac{\partial U}{\partial X_{2}}\right)=0
$$

where $\rho$ is a function of the first derivatives of $U$, can be linearised by a hodograph transformation [9]. 
3. Development of modified approximations near the singularity

Consider, for example, the two-dimensional, elliptic case of (1.3) and assume that it is of such a form that it can be reduced using transformations (2.14) and (2.17) to the form

$$
\frac{\partial^{2} u}{\partial x^{2}}+\frac{\partial^{2} u}{\partial y^{2}}-g(x, y) u-f(x, y)=0
$$

where the transformation between $U$ and $u$ is given by say,

$$
\mathrm{U}=\mathrm{L}[\mathrm{u}] \text {. }
$$

Assume that an analytical solution of the form (2.8), valid near the boundary singularity at the point 0 , has been found. Taking the first $\mathrm{N}$ terms of (2.8) an approximation for $\mathrm{u}$, valid near 0 , is

$$
\mathrm{u}^{*}(\rho, \theta)=\phi_{0}(\rho, \theta)+\sum_{\mathrm{i}=1}^{\mathrm{N}} \mathrm{c}_{\mathrm{i}} \phi_{\mathrm{i}}(\rho, \theta)
$$

and the corresponding approximation for $U$ is defined by

$$
\mathrm{U}^{*}(\rho, \theta)=\mathrm{L}\left[\mathrm{u}^{*}(\rho, \theta)\right] \equiv \psi_{0}(\rho, \theta)+\sum_{\mathrm{i}=1}^{\mathrm{N}} \mathrm{c}_{\mathrm{i}} \psi_{\mathrm{i}}(\rho, \theta)
$$

Instead of applying standard finite-difference or finite element approximations throughout the entire region a neighbourhood $\mathrm{N}(0)$ near the singularity is chosen and, for points in $\mathrm{N}(0)$, modified approximations for $u$ and its derivatives in (3.1) are developed which take into account the nature of the singularity. The derivatives are approximated using the standard differential relations

$$
\begin{aligned}
& \frac{\partial^{2} u}{\partial x^{2}}=\cos ^{2} \theta \frac{\partial^{2} u}{\partial \rho^{2}}-\frac{\sin 2 \theta}{\rho} \frac{\partial^{2} u}{\partial \rho \partial \theta}+\frac{\sin ^{2} \theta}{\rho^{2}} \frac{\partial^{2} u}{\partial \theta^{2}}+\frac{\sin ^{2} \theta}{\rho^{2}} \frac{\partial u}{\partial \rho}+\frac{\sin 2 \theta}{\rho^{2}} \frac{\partial u}{\partial \theta} \\
& \frac{\partial^{2} u}{\partial y^{2}}=\sin ^{2} \theta \frac{\partial^{2} u}{\partial \rho}-\frac{\sin 2 \theta}{\rho} \frac{\partial^{2} u}{\partial \rho \partial \theta}+\frac{\cos ^{2} \theta}{\rho^{2}} \frac{\partial^{2} u}{\partial \theta^{2}}+\frac{\cos ^{2} \theta}{\rho} \frac{\partial u}{\partial \rho} \frac{\sin 2 \theta}{\rho^{2}} \frac{\partial u}{\partial \theta}
\end{aligned}
$$


where approximations for the $\rho$ and $\theta$ derivatives are obtained by differentiating (3.3). Thus approximations for (3.5) and (3.6) can be expressed as

$\left.\begin{array}{rl}\frac{\partial^{2} u^{*}}{\partial x^{2}} & =\sum_{i=1}^{N} c_{i} w_{i}(\rho, \theta) \\ \frac{\partial^{2} u^{*}}{\partial y^{2}} & =\sum_{i=1}^{N} c_{i} w_{i}(\rho, \theta)\end{array}\right\}$ with truncation error $0\left(\rho^{M-2}\right)$

where $M$ is the highest power of $\rho$ in the $\phi(\rho, \theta)$ of $(3.3)$.

The $w_{i}, w_{i}^{\prime}$ are readily found by following the above steps and the symmetry in (3.5) and (3.6) leads to symmetry between the $\mathrm{w}_{\mathrm{i}}$ and

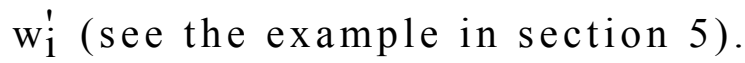

The $\mathrm{N}$ unknown constants $\mathrm{c}_{\mathrm{i}}$ are approximated by fitting (3.4) to the set of $U$ values $\left\{U_{j}: j=1,2, \ldots, N\right\}$ at $N$ points in and near $\mathrm{N}(0)$. More than $\mathrm{N}$ points can be used if a best fit (e.g. least squares) technique is used. For each point $\mathrm{n}$, say, in $\mathrm{N}(0)$ the same set of points for the $\left\{U_{j}\right\}$ values can be used. In this case the set usually consists of points just outside $\mathrm{N}(0)$, - called 'far' points, Motz [21]. Alternatively, a different set of points for each point $n$ can be used. In this case the set usually consists of points surrounding the point $n$, Bell and Crank [6]

The proposed method is a variation of the latter type in that a different set of points is used for each $x$ and $y$ derivative for each point $\mathrm{n}$. The method is illustrated by developing a modified five-point formula for the typical point 1 in $\mathrm{N}(0)$, see Figure 1.

Figure 1

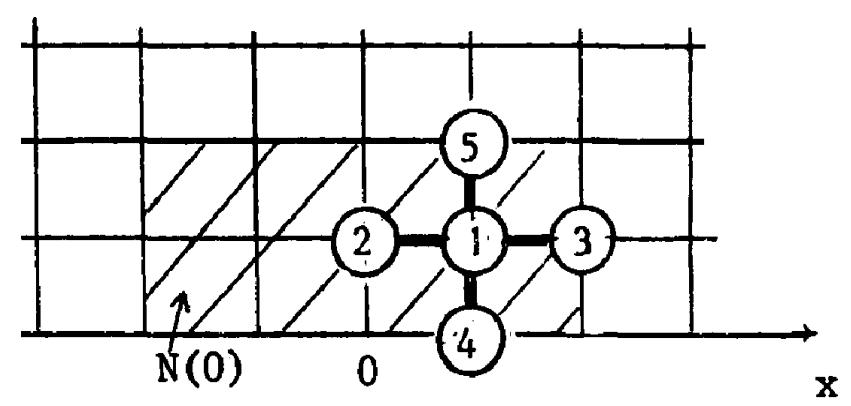


To construct a five-point formula the approximations (3.3) and (3.4) are used with $\mathrm{N}=3$. Referring to Figure 1, and denoting $U_{j}^{*}, \rho_{j}, \theta_{j}$ to be the corresponding $U^{*}, \rho, \theta$ values at the points $\mathrm{j}=1$ to 5 , then the most obvious choice of points to approximate the unknown constants $c_{i}$ in (3.7) for $\left.\frac{\partial^{2} u^{*}}{\partial x^{2}}\right|_{\text {point } 1}$ is to use

points $\mathrm{j}=1,2,3$ in the horizontal direction. Using (3.4) this gives the following three equations for the $c_{i}$

$$
U_{j}^{*}=\psi_{0}\left(\rho_{j}, \theta_{j}\right)+\sum_{i=0}^{n} c_{i} \psi_{i}\left(\rho_{j}, \theta_{j}\right), j=1,2,3,
$$

whose solution can be denoted by

$$
c_{i}=A_{i}+B_{i} U_{2}^{*}+C_{i} U_{1}^{*}+D_{i} U_{3}^{*}, \quad i=1,2,3 .
$$

Similarly, for the $c_{i}$ in (3.8) for $\left.\frac{\partial^{2} u}{\partial y^{2}}\right|_{\text {point } 1}$, point 4,1 and 5

in the vertical direction are used and the solution is denoted by

$$
\mathrm{c}_{\mathrm{i}}=\mathrm{A}_{\mathrm{i}}^{\prime}+\mathrm{B}_{\mathrm{i}}^{\prime} \mathrm{U}_{4}^{*}+\mathrm{C}_{\mathrm{i}}^{\prime} \mathrm{U}_{1}^{*}+\mathrm{D}_{\mathrm{i}}^{\prime} \mathrm{U}_{5}^{*}, \quad \mathrm{i}=1,2,3 .
$$

Substituting (3.10) and (3.11) in (3.7) and (3.8), and using (3.3) with the $c_{i}$ given by (3.10) for the term $g(x, y) u$ in (3.1), gives the following five-point approximation for (3.1) at the typical point 1 in $\mathrm{N}(0)$

$$
\frac{\partial^{2} u^{*}}{\partial x^{2}}+\frac{\partial^{2} u^{*}}{\partial y^{2}}-g u^{*}-f=e_{2} U_{2}^{*}+e_{3} U_{3}^{*}+e_{4} U_{4}^{*}+e_{5} U_{5}^{*}+e_{1} U_{1}^{*}+e_{6}=0
$$

Where

$$
\left.\begin{array}{l}
\mathrm{e}_{1}=\sum_{i=1}^{3}\left[\mathrm{C}_{\mathrm{i}}\left(\mathrm{w}_{\mathrm{i}}-\mathrm{g} \phi_{\mathrm{i}}\right)+\mathrm{w}_{\mathrm{i}}^{\prime} \mathrm{C}_{\mathrm{i}}^{\prime}\right], \quad \mathrm{e}_{2}=\sum_{\mathrm{i}=1}^{3} \mathrm{~B}_{\mathrm{i}}\left(\mathrm{w}_{\mathrm{i}}-\mathrm{g} \phi_{\mathrm{i}}\right), \\
\mathrm{e}_{3}=\sum_{\mathrm{i}=1}^{3} \mathrm{D}_{\mathrm{i}}\left(\mathrm{w}_{\mathrm{i}}-\mathrm{g} \phi_{\mathrm{i}}\right), \mathrm{e}_{4}=\sum_{\mathrm{i}=1}^{3} \mathrm{w}_{\mathrm{i}}^{\prime} \mathrm{B}_{\mathrm{i}}^{\prime}, \\
\mathrm{e}_{5}=\sum_{\mathrm{i}=1}^{3} \mathrm{w}_{\mathrm{i}}^{\prime} \mathrm{D}_{\mathrm{i}}^{\prime}, \quad \mathrm{e}_{6}=\sum_{\mathrm{i}=1}^{3}\left[\mathrm{~A}_{\mathrm{i}}\left(\mathrm{w}_{\mathrm{i}}-\mathrm{g} \phi_{\mathrm{i}}\right)+\mathrm{A}_{\mathrm{i}}^{\prime} \mathrm{w}_{\mathrm{i}}^{\prime}\right]-\mathrm{g} \phi_{0}-\mathrm{f} .
\end{array}\right\}
$$


14.

The functions $w_{i}, w_{i}^{\prime}, \phi_{i}, g$ and $f$ are evaluated at the point $\left(\rho_{1}, \theta_{1}\right)$. If the original differential equation (1.3) is equivalenced (via the zero right-hand side) to the reduced equation (3.1) then (3.12) represents a five-point approximation to the original equation.

The above technique of horizontal and vertical derivative replacement is based on that used by Bell and Crank [6] but is a generalisation in that

(i) both derivatives are treated,

(ii) the approximations (3.7) and (3.8) are written in a general way so that the neighbouring points chosen to approximate the $\mathrm{c}_{\mathrm{i}}$ need not lie on the same horizontal or vertical line.

Generalisation (ii) is useful in developing higher-order, multi-point modified approximations by varying the number of terms included in the truncated series expansion and the set of neighbouring points used for any one point in $\mathrm{N}(0)$. The neighbourhood $\mathrm{N}(0)$ can include points away from 0 as long as the approximations (3.3) and (3.4) remain valid (this may be checked as described in Motz [21]). The optimum size of $\mathrm{N}(0)$ can be determined by comparison of the discretisation error in the standard approximations used outside $\mathrm{N}(0)$ with the truncation error in (3.7) and (3.8), see section 5 .

The five-point approximation (3.12) could be incorporated in either a finite-difference or finite element scheme. An equivalent finite element approximation can be developed by the use of equations similar to $(3.5)-(3.8)$ for approximations to $\frac{\partial u^{\cdot}}{\partial x^{\prime}} \frac{\partial u}{\partial y}$ in the variational formulation corresponding to (3.1). For example, in the solution of the problem of Motz considered in section 5, both Barnhill and Whiteman [3] and Wait and Mitchell [35] used the variational functional

$$
\mathrm{I}[\mathrm{u}]=\iint\left[\left[\frac{\partial \mathrm{u}}{\partial \mathrm{x}}\right]^{2}+\left[\frac{\partial \mathrm{u}}{\partial \mathrm{y}}\right]^{2}\right] \mathrm{dR},
$$

where $\mathrm{R}$ is the closed region under consideration. 
15.

Five-point 'molecules' differing from the one given in Figure 1 are needed for points in $\mathrm{N}(0)$ which involve the boundary. Two problems can arise, the first is that some of the five points required may, depending on the geometry of the problem, lie outside the region of solution, and the second is that the values of $\psi_{i}(\rho, \theta)$ at the point $\left(\rho_{\mathrm{j}}, \theta_{\mathrm{j}}\right)$ may all be zero (e.g. if in $(2.5), \theta=\omega=\pi$ ) so that solutions to (3.9) for the $c_{i}$ cannot be found. The remedy for both problems is simply to choose alternative points near $\left(\rho_{\mathrm{j}}, \theta_{\mathrm{j}}\right)$ giving a new five-point molecule, e.g. see section 5, Figures 4-6.

\section{A. Discussion and extensions of the modified approximations}

An alternative way of approximating $u^{*}$ in (3.3) is to split it into the form

$$
u^{*}(\rho, \theta)+\sum_{i=1}^{n} c_{i} S_{i}(\rho, \theta),
$$

where the function $\eta$ represents the well-behaved part and the functions $S_{i}$ represent the singular terms only in the series expansion (3.3). In this case fewer terms in the series in (4.1) are needed than in (3.3), usually one or two singular terms are sufficient, and this is advantageous in situations where it is difficult to obtain more than one or two terms in (3.3). This approach was first proposed by Woods [42] and has been extended to finite-difference and finite element methods by Emery and Segedin [14,15], Benzley [7],

Both the proposed method and the alternative Woods approach can readily be extended to the parabolic, time-dependent, case since the form of the boundary singularity depends on the space co-ordinates rather than the time co-ordinate. Thus seeking a solution in the form (2.12) or, equivalently, using the exponential transformation (2.17) in time, one finds that the time-dependent form of (3.3) can be expressed as

$$
\mathrm{u}^{*}(\rho, \theta, \mathrm{t})=\phi_{0}(\rho, \theta, \mathrm{t})+\sum_{\mathrm{i}=1}^{\mathrm{N}} \mathrm{c}_{\mathrm{i}}(\mathrm{t}) \phi_{\mathrm{i}}(\rho, \theta)
$$


16.

and so the above methods can be applied with the $c_{i}$ being re-evaluated at each time level, see Bell and Crank [4-6].

Equations of higher order than (1.3) could be treated in a similar way by considering higher order differential relations to (3.5) and (3.6). The biharmonic equation used in elasticity problems, e.g. in two dimensions

$$
\nabla^{4} U=\frac{\partial^{4} U}{\partial X_{1}^{4}}+2 \frac{\partial^{4} U}{\partial X_{1}^{2} X_{2}^{2}}+\frac{\partial^{4} U}{\partial X_{2}^{4}}=0
$$

can be formulated, using Southwell's stress function formulation, as two second order equations and thus the techniques of section 3 can be used. Boundary singularities for such problems have been treated by Bernal and Whiteman [8], Zak [43]. Emery and Segedin [14] have given the necessary singular functions for (4.3).

The theory of sections 2 and 3 has been developed for a singularity at the point 0 where two straight line segments $\theta=0$ and $\theta=\omega$ meet and would need modification for points involving curved segments unless conformal transformations are first used to transform the curved segments to straight lines. Unless this is done the curved segments will have to be approximated by straight lines (only for a short distance from 0 ) or the local analytic form and derivative approximations will have to take into account the curvature. A particular example of such curved segments occurs at the separation point between the fixed and free boundaries in problems concerning seepage through a dam [1] and cavitational flow past a disc [18].

The line integral

$$
\mathrm{I}=\oint_{\Gamma}\left[\frac{\partial \mathrm{U}}{\partial \mathrm{n}}\right]_{\Gamma} \mathrm{d} 1,
$$

where $\Gamma$ represents part of the boundary and includes the boundary singularity is often required, e.g. for the evaluation of the flux in diffusion problems, Crank and Furzeland [11] or for the measurement of capacitance in potential problems, Duncan [13]. Large errors 
near the singularity can be avoided if the analytical form (3.3) is used in the numerical quadrature of (4.4) near the singularity, see Crank and Furzeland.

Modified approximations such as (3.12) can be developed by heat balance methods, see Bell and Crank [4-6], or by the analogous integration method of Varga [33],

5 Application of the method to the problem of Motz and Woods

The problem of Motz [21] requires the solution of Laplace's equation in a rectangle with a slit, i.e. a re-entrant corner of internal angle $2 \Pi$ (see Figure 2). It has been treated by many authors to demonstrate the effectiveness of their singularity treatments. Woods [42] gave an alternative formulation based on the fact that u - 500 is antisymmetric about the line BE containing the slit and, by imposing the boundary condition $u=500$ on E0, only needed to consider the top half of the rectangle (Figure 3 ). It is in this form that the problem is treated in the literature [3,19,22,23,32,35-39].

Figure 2

Figure 3

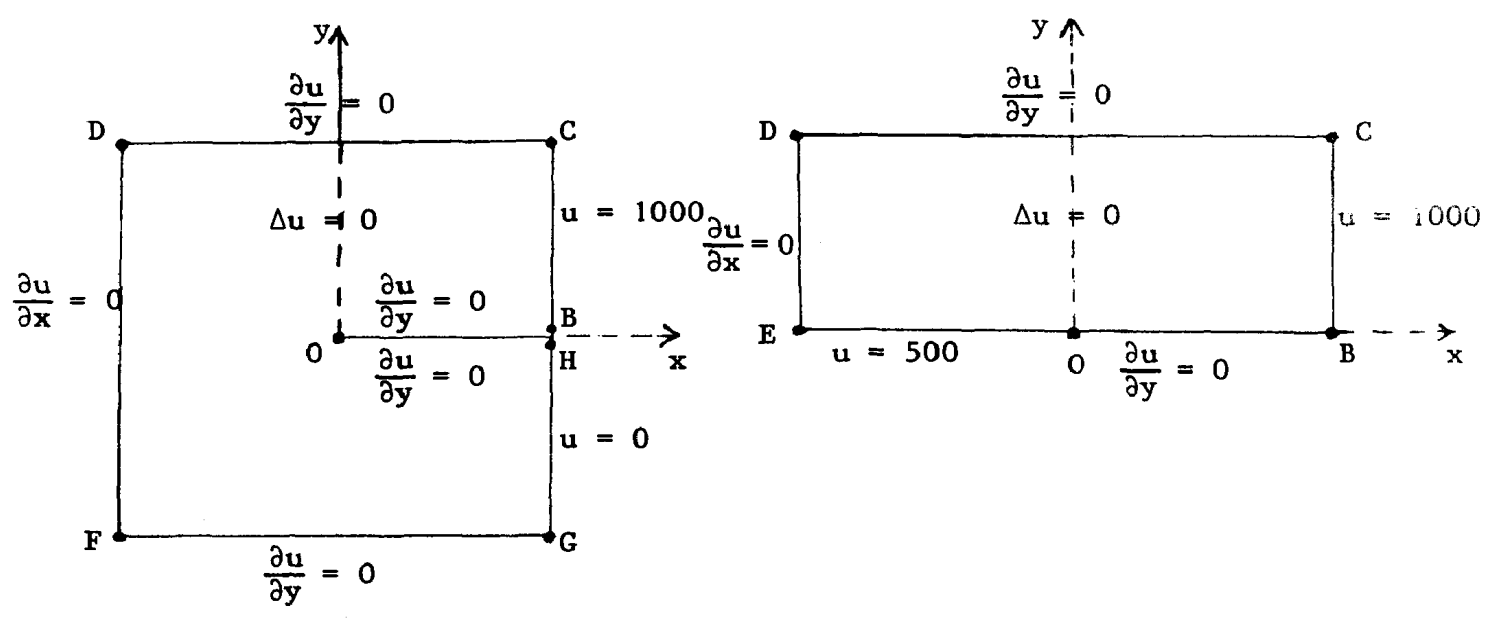

Examples of such singularities occur in potential problems concerning transmission lines with microstrips, Daly [12], and in diffusion problems with narrow band type electrodes, Saito [27]. 
18.

Since the governing Laplacian equation is already in the simple form (1.2) with $\mathrm{g}=\mathrm{f}=0$ there is no need for a transformation of co-ordinates or unknown function. For an application of the method to a problem where such a transformation is necessary see Crank and Furzeland [11] who treat problems of the form (2.21). In the Woods formulation the singularity is regarded as resulting from the mixed boundary conditions

$$
\frac{\partial \mathrm{u}}{\partial \mathrm{y}}=0, \mathrm{x}>0 ; \mathrm{u}=500, \mathrm{x} \leq 0 \text { on } \mathrm{y}=0
$$

rather than being due to the re-entrant corner of $2 \Pi$ with homogeneous Neumann conditions on both arms. A separable-variable solution of the form (2.2) in the polar co-ordinate form of Laplace's equation (see (2.1) with $\mathrm{g}=\mathrm{f}=0$ ) yields the local analytic form

$$
u=500+\sum_{i=1}^{\infty} c_{i} \rho^{(2 i-1) / 2} \cos \frac{(2 i-1)}{2} \theta
$$

The problem of Figure 3 is scaled by setting

(i) $\mathrm{v}=\mathrm{u}-500$,

(ii) $\mathrm{B}=(1,0) ; \mathrm{c}=(1,1) ; \mathrm{D}=(-1,1) ; \mathrm{E}=(-1,0)$,

and standard five-point finite-difference approximations are used for the discretised region of Figure 7 with $\delta x=\delta y=h$. To enable comparisons the choice of discretisation follows that of Motz and Woods, although this choice results in unequal intervals near the edges and so the discretisation error is only $0(\mathrm{~h})$ for these points. For points involving the Neumann boundary condition the usual central difference approximations for the derivatives are used in conjuction with fictitious points. Figure 7 compares the numerical solution obtained using these standard finitedifference approximations with the highly accurate results produced by the conformal transformation method of Papamichael and Whiteman [23]. The results show that a high degree of inaccuracy occurs near the singularity and illustrate the fact that inaccuracies spread throughout the entire region. 
Instead of applying the standard finite-difference approximations throughout the entire region, a neighbourhood $\mathrm{N}(0)$ near the singularity is chosen and, for points in $\mathrm{N}(0)$ modified approximations are developed which take into account the nature of the singularity given by (5.2). Following section 3 the derivative approximations (3.7) and (3.8) with $\mathrm{N}=3$ lead to the modified five-point approximation (3.12) where we have

$$
\left.\begin{array}{l}
\mathrm{g}=\mathrm{f}=0, \quad \mathrm{e}_{6}=0, \\
\mathrm{w}_{1}=-\frac{\cos \frac{3}{2} \theta}{4 \rho^{3 / 2}} ; \quad \mathrm{w}_{2}=\frac{3 \cos \frac{1}{2} \theta}{4 \rho^{\frac{1}{2}}} ; \mathrm{w}_{3}=\frac{15 \rho^{\frac{1}{2}} \cos \frac{1}{2} \theta}{4} ; \\
\mathrm{w}_{\mathrm{i}}^{\prime}=-\mathrm{w}_{\mathrm{i}}, \quad \mathrm{i}=1,2,3 .
\end{array}\right\}
$$

The approximate size of $\mathrm{N}(0)$ can be determined by noting that the discretisation error in the standard five-point approximations to Laplace's equation is $0\left(\mathrm{~h}^{2}\right)$, see $(1.1)$, whereas it is easily verified that the modified approximations based on (3.7) and (3.8) with $\mathrm{N}=3$ contain a truncation error of $0\left(\rho^{3 / 2}\right)$. Thus application of the modified approximations is advantageous as long as the truncation error does not exceed the discretisation error. An approximate rule is then to choose $\mathrm{N}(0)$ such that the maximum $\rho$ value in $\mathrm{N}(0), \rho_{\max }$ say, is such that $\rho_{\max }^{3 / 2}$. is of the same order of magnitude as $h^{2}$. Practical experience suggests that $\rho_{\max }<5 \mathrm{~h}^{2}$ is a useful guide. In practice only a few points in $\mathrm{N}(0)$ are needed.

Five-point 'molecules' differing from that given in Figure 1 are needed for points in $\mathrm{N}(0)$ which involve the boundary. Points to the right of 0 on $\mathrm{j}=0$ do not have a point at the $\mathrm{j}-1$ level, necessitating a different molecule, e.g. Figure 4. Points to the left of 0 on $j=1$ involve points on $j=0$ for which $\theta=\pi$. The fact that $\theta=\pi$ means that, by comparing (5.2) with (3.9), each of the $\psi_{i}(\rho, \theta)$ are zero and thus solutions to (3.10) cannot be found. A suggested alternative is given in Figure5. The first point on the right of 0 on $\mathrm{j}=0$ involves both the above problems and a suggested molecule is given in Figure 6. The general form of the modified approximations allows for any combination of five neighbouring points provided $\theta \neq \pi$. 

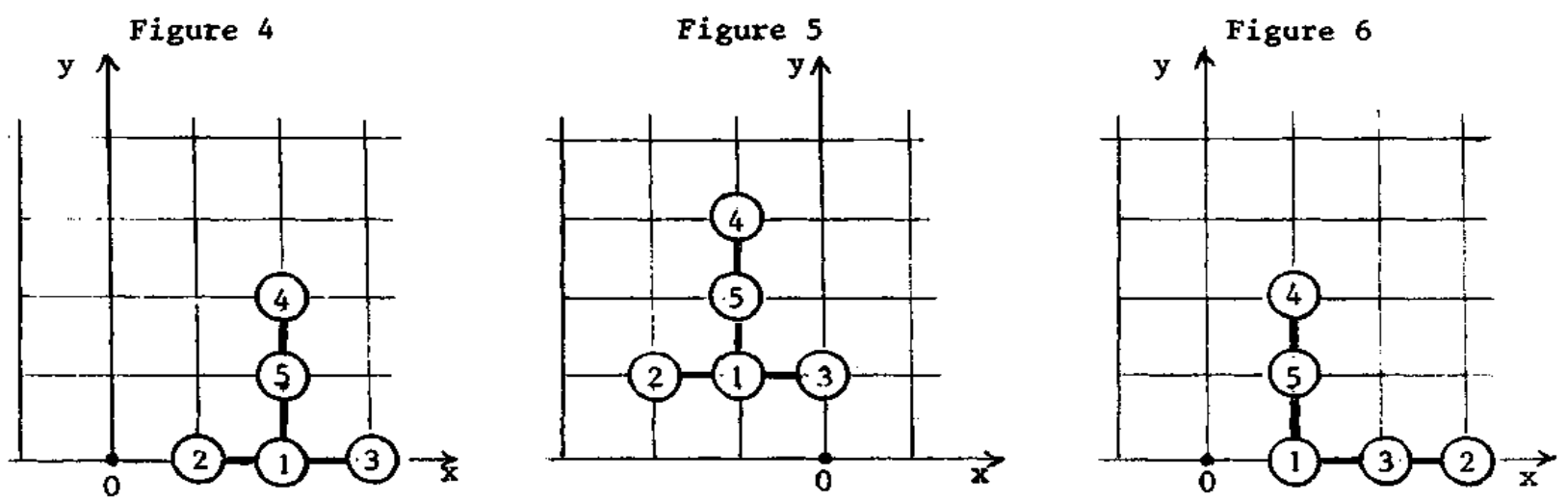

Referring to Figure 8 , the four immediately neighbouring points $\mathrm{P}_{1}$, $\mathrm{P}_{2}, \mathrm{P}_{3}$ and $\mathrm{P}_{4}$ around 0 are chosen for $\mathrm{N}(0)$ since here $\rho_{\max }^{3 / 2} \approx 0.26$ and $\mathrm{h}^{2} \approx 0.08$. This choice of points is similar to that used by Motz and Woods and enables comparisons to be made with their results. Modified approximations of the form (3.12) and (5.5) are applied at points inside $\mathrm{N}(0)$ using the suggested molecules of Figures 4-6 where appropriate. Standard finitedifference approximations are used for points outside $\mathrm{N}(0)$. The results obtained are comparable with those of Motz and Woods and give good agreement with the conformal transformation values of Papamichael and Whiteman [23].

The values of the $e_{i}$ in (3.12) are given in Figures 9-12 for the points $\mathrm{P}_{1}-\mathrm{P}_{4}$ with $\mathrm{h}=2 / 7$. The values given have been scaled so that $\mathrm{e}_{1}=-4.0000$. 


\section{$\underline{\text { References }}$}

1. J. Aitchison, Numerical treatment of a singularity in a free boundary problem, Proc. R. Soc. Lond. A.,330 (1972),573-580.

2. I. Babuska and A.K. Aziz, The finite element method for non-smooth domains and coefficients, in "The Mathematical Foundations of the Finite Element Method with Applications to Partial Differential Equations" (A.K. Aziz, Ed.), Academic Press, New York (1972), 265-283.

3. R.E. Barnhill and J.R.Whiteman, Error analysis of finite element methods with triangles for elliptic boundary value problems, in "The Mathematics of Finite Elements and Applications" (J.R. Whiteman, Ed.), Academic Press, London, (1973), 83-112.

4. G.E. Bell, Diffusion in composite media and associated mathematical problems, Ph.D. thesis, Brunel University, (1973).

5. G.E. Bell and J. Crank, A method of treating boundary singularities in time-dependent problems, J. Inst. Maths. Applies. 12 (1973), 37-48.

6. G.E. Bell and J. Crank, A simple finite-difference modification for improving accuracy near a corner in heat flow problems, Int. J. Num. Meth. Engng. 10 (1976), 827-832.

7. S.E. Benzley, Representation of singularities with isoparametric finite elements, Int. J. Num. Meth. Engng. 8 (1974), 537-545.

8. M.J.M. Bernal and J.R. Whiteman, Numerical treatment of biharmonic boundary value problems with re-entrant boundaries, Computer J.13 (1970), 87-91.

9. L. Bers, Results and conjectures in the mathematical theory of subsonic and transonic gas flows, Comm. Pure Appl. Maths.7(1954), 79-104; see also $105-148$.

10. R. Courant and D. Hilbert, "Methods of Mathematical Physics", vol.11, Interscience, London, (1962).

11. J. Crank and R.M. Furzeland, The treatment of boundary singularities in axially symmetric problems containing discs, J. Inst. Maths. Applics, (to appear).

12. P. Daly, Singularities in transmission lines, in "The Mathematics of Finite Elements and Applications" (J.R. Whiteman, Ed.), Academic Press, London, (1973), 337-350.

13. J.W. Duncan, The accuracy of finite-difference solutions of Laplace's equation, IEEE Trans. MTT-15 (1967), 575-582.

14. A.F. Emery and C.M. Segedin, A numerical technique for determining the effect of singularities in finite-difference solutions illustrated by application to plane elastic problems, Int. J. Num. Meth. Engng. 6 (1973), 367-380. 
15. A.F. Emery, The use of singularity programming in finite-difference and finite-element computations of temperature, Trans. ASME (C) 95 (1973), 344-351.

16. L. Fox, Some experiments with singularities in linear elliptic partial differential equations, Proc. Roy. Soc. Lond. A. 323 (1971), 179-190.

17. L. Fox and R. Sankar, Boundary singularities in linear elliptic differential equations, J. Inst. Maths. Applies. 5 (1969), 340-350.

18. L. Fox and R. Sankar, The regula-falsi method for free boundary problems, J. Inst. Maths. Applics. 12 (1973), 49-54.

19. J.A. Gregory and J.R. Whiteman, Local mesh refinement with finite elements for elliptic problems. Technical Report TR/24, Department of Mathematics, Brunel University (1974).

20. R.S. Lehman, Developments at an analytic corner of solutions of elliptic partial differential equations, J. Maths. Mech., 8 (1959), 727-760.

21. H. Motz, The treatment of singularities of partial differential equations by relaxation methods, Quart. App1. Math. 4 (1946), 371-377.

22. N. Papamichael and J.B. Rosser, A power series solution for a harmonic mixed boundary value problem, Technical Report TR/35, Department of Mathematics, Brunel University (1973).

23. N. Papamichael and J.R. Whiteman, Treatment of harmonic mixed boundary value problems by conformal transformation methods, Z. Angew Math. Phys. 23 (1972), 655-664.

24. N. Papamichael and J.R. Whiteman, A numerical conformal transformation method for harmonic mixed boundary value problems in polygonal domains, Z. Angew Math. Phys. 24 (1973), 304-316.

25. V.Z. Parton, The axially symmetric temperature problem for the space with a disk-shaped crack, J. Appl. Maths. Mech. 36 (1972), 104-111.

26. P.M. Riz, in "Linear Equations of Mathematical Physics" (S.G. Mikhlin, ed.), Holt, Rinehart and Winston, New York, (1967), 1-19.

27. Y. Saito, A theoretical study on the diffusion current at the stationary electrodes of circular and narrow band types, Rev. Polarography (Japan) 15 (1968), 177-187.

28. R. Sankar, Numerical solution of differential equations, D.Phil. thesis, Oxford (1967).

29. A. Sideridis, Brunel University, private communication (1976). 
30. I.N. Sneddon, "Mixed Boundary Value Problems in Potential Theory", Wiley, New York, (1966).

31. G.T. Symm, Treatment of singularities in the solution of Laplace's equation by an integral equation method, NPL Report NAC31 (1973).

32. R.W. Thatcher, Singularities in the solution of Laplace's equation in two dimensions, J. Inst. Maths. Applics. 16 (1975), 303-319.

33. R.S. Varga, "Matrix Iterative Analysis", Prentice-Hall, London, (1962), 181-193.

34. E.A. Volkov, The removal of singularities in the solution of boundary value problems for the Laplace equation in a region with a smooth boundary, USSR Comp. Math. Math. Phys. 3 (1963), $139-152$.

35. R.Wait and A.R. Mitchell, Corner singularities in elliptic problems by finite element methods, J. Comp. Phys. 8 (1971), 45-52.

36. J.C. Webb and J.R. Whiteman, Convergence of finite-difference techniques for a harmonic mixed boundary value problem, B.I.T. 10 (1970), 366-374.

37. J.R. Whiteman, Singularities due to re-entrant corners in harmonic boundary value problems, Technical Report No.829, Mathematics Research Center, University of Wisconsin, Madison (1967).

38. J.R. Whiteman, Numerical solution of a harmonic mixed boundary value problem by the extension of a dual series method, Q.J. Mech. Appl. Math. 23 (1970), 449-455.

39. J.R. Whiteman, in Vol.2 "Proceedings of International Symposium on Finite Elements in Flow Problems" (editors: R. Gallagher, J.T. Oden, C. Taylor and O.C. Zienkiewicz), Wiley, London, (1974), $101-119$.

40. N.W. Wigley, Asymptotic expansions at a corner of solutions of mixed boundary value problems, J. Math. Mech. 13 (1964), 549-576.

41. N.W. Wigley, On a method to subtract off a singularity at a corner for the Dirichlet or Neumann problem, Math. Comp. 23 (1969), 395-401.

42. L.C. Woods, The relaxation treatment of singular points in Poisson's equation, Q.J. Mech. Appl. Math. 6 (1953), 163-189.

43. A.R. Zak, Elastic analysis of cylindrical configurations with stress singularities, J. Appl. Mech., Trans. ASME (E) 39 (1972), 501-506; see also discussion p.1169. 
igure 7

$$
\frac{\partial u}{\partial y}=0 \uparrow^{y}
$$

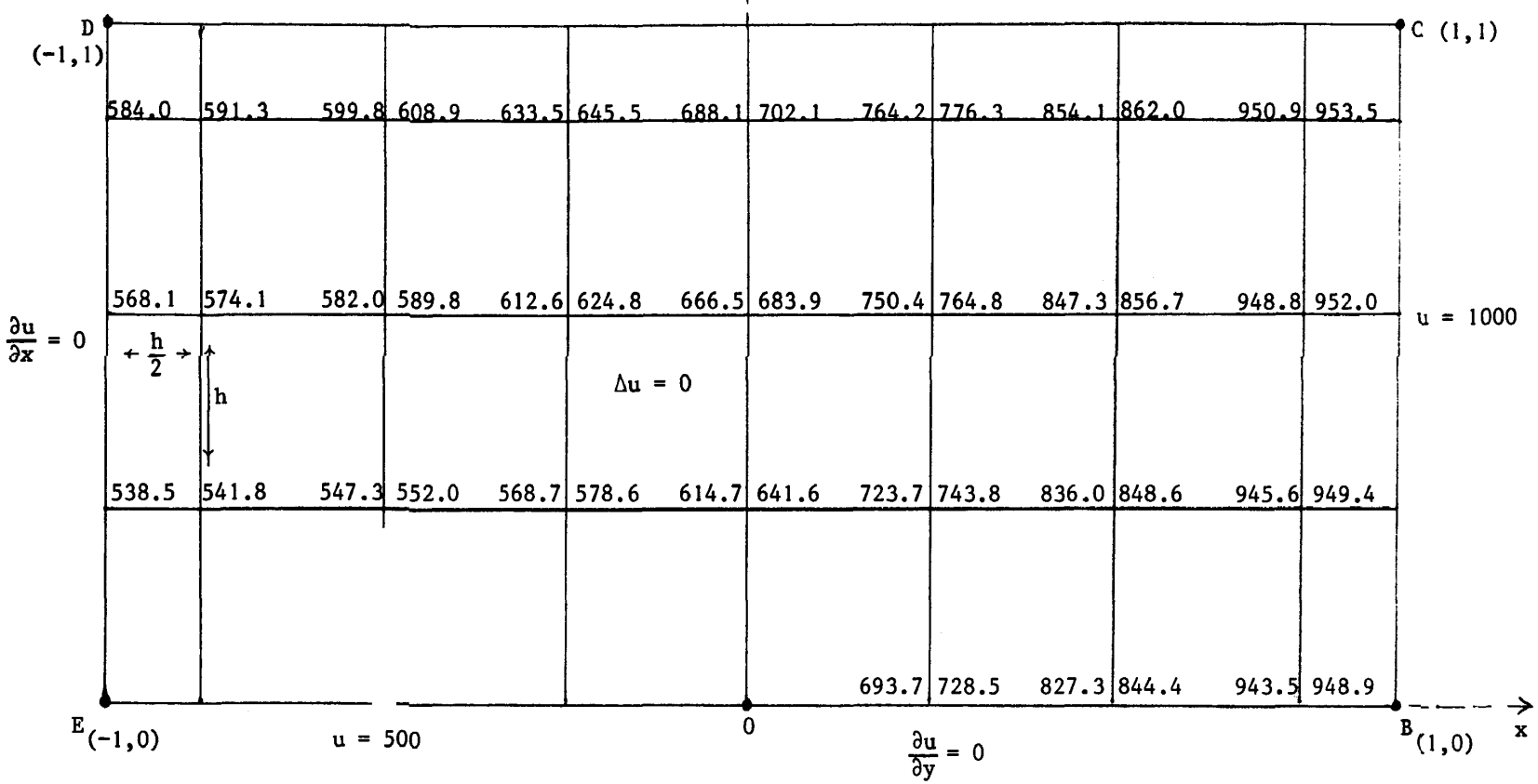

$D C=E B=2$ units

$\mathrm{DE}=\mathrm{BC}=1$ unit

$\underline{\mathrm{KEY}}$

$\delta x=\delta y=2 / 7$ (except near $\mathrm{ED}, \mathrm{DC}, \mathrm{CB}$ )

Finite-difference solution, no singularity treatment
Conformal transformation solution, PAPMICHAEL and WHITEMAN [23] 
igure 8

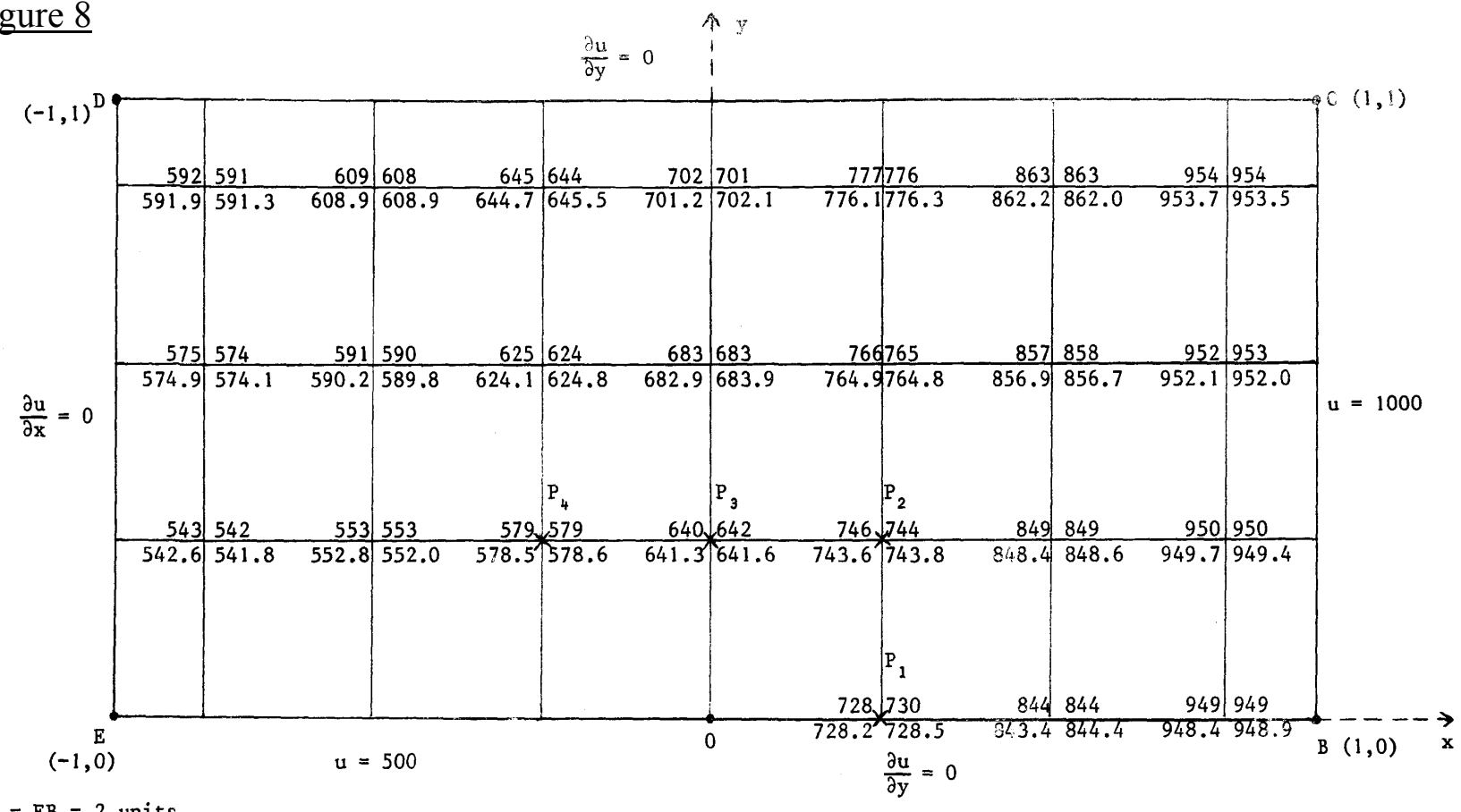

$\mathrm{DC}=\mathrm{EB}=2$ units

$\mathrm{DE}=\mathrm{BC}=1$ unit

$\delta x=\delta y=2 / 7$ (except. near $E D, D C, C B$ )

$\underline{\text { KEY }}$

\begin{tabular}{|c|c|c|}
\hline KEY & $\begin{array}{l}\text { MOTZ method using } \\
\text { antisymmetry } \\
\text { Whiteman }[37]\end{array}$ & $\begin{array}{l}\text { Woods } \\
\text { method [42] }\end{array}$ \\
\hline & $\begin{array}{l}\text { CRANK and FURZELAND } \\
\text { modified formulae } \\
\text { at } p_{\text {i to }} P_{4} \text {. The } u \\
\text { valus are from }+500 \text {, } \\
\text { se }=(3,3) \text {. }\end{array}$ & $\begin{array}{l}\text { PAPAMICHAEL and WHITEMAN } \\
\text { conformal transformation } \\
\text { nethod }[23]\end{array}$ \\
\hline
\end{tabular}

t These values are used since they take into account the antisymmetry whereas Motz's work [21] did not.

$\frac{\partial u}{\partial y}=0$ 
26.

Figure 9

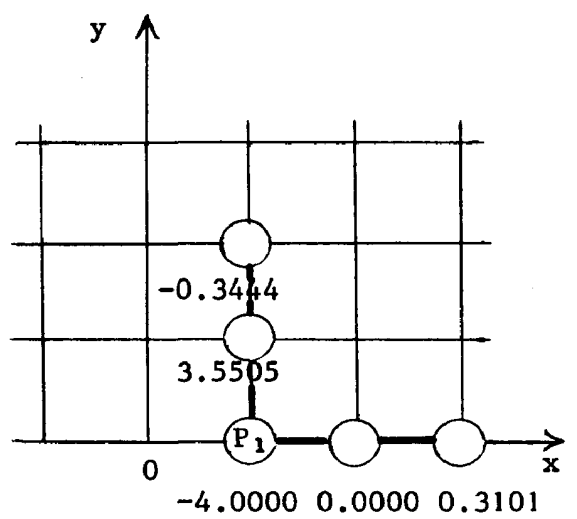

Figure 11

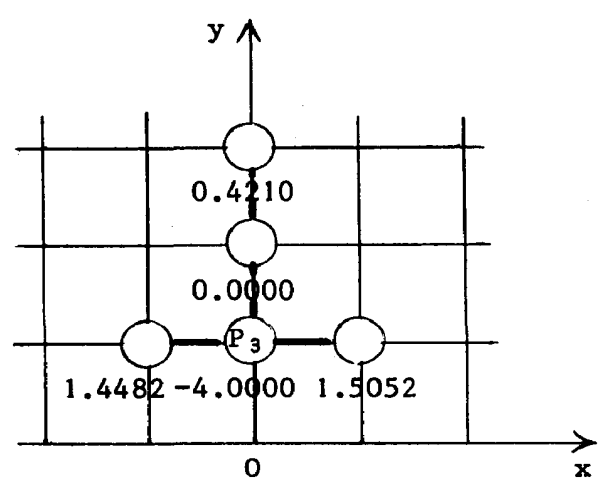

Figure 10

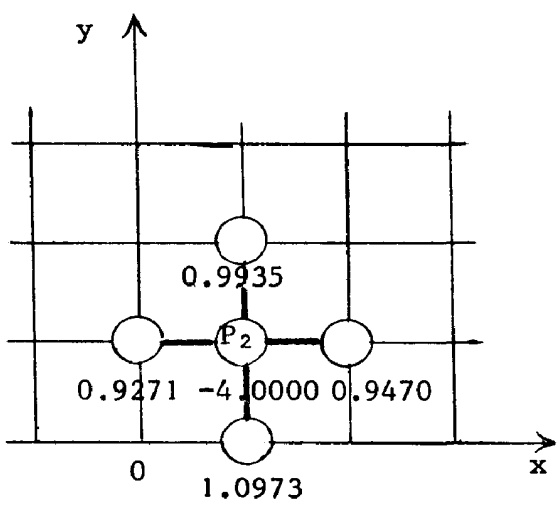

Figure 12

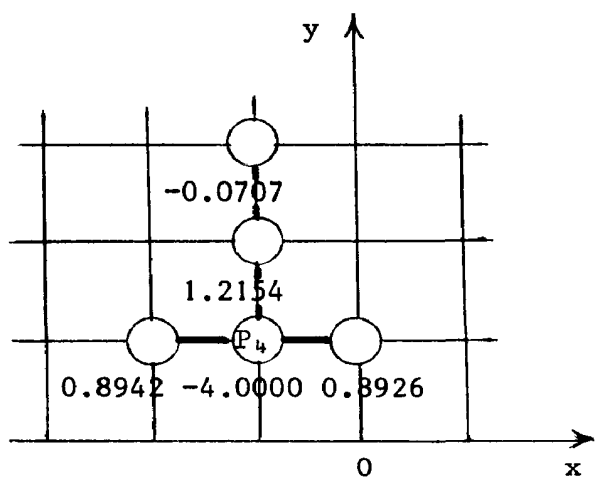

N.B. The above $e_{i}$ values are for $v=u-500$ values, see (5.3). 